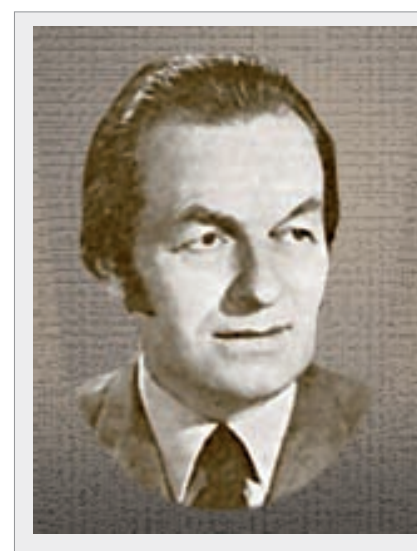

Principales dates

16 janvier 1919 - Naissance à Nowy Targ (Pologne)

1954 Création du Laboratoire de microscopie optique

1965

Premier microscope Zeiss à contraste interférentiel différentiel

1973

Abbe Medal de la State Microscopical Society of Illinois

1995

Gold medal de la SPIE

17 février 1997 - Mort à Antony (France)

\title{
Georges (Jerzy) Nomarski
}

Riad Haidar

haidar@onera.fr

Physicien franco-polonais, ingénieur opticien et théoricien de talent, Georges (Jerzy) Nomarski est principalement connu pour ses contributions à la microscopie à contraste de phase, et notamment l'invention du microscope à contraste interférentiel différentiel qu'il développe dans les années 1950.

$\mathrm{J}$ erzy Nomarski naît le 6 janvier 1919 dans la ville polonaise de Nowy Targ, dans la voïvodie (région) de Petite-Pologne, à une centaine de kilomètres de Cracovie, au sud du pays. Il est formé à la prestigieuse École polytechnique de Varsovie. À 20 ans, il se passionne pour l'optique et envisage une carrière de physicien... Maisl'histoire de la Pologne bascule. En septembre 1939, Varsovie capitule sous les assauts conjugués de la Wehrmacht et de l'Armée rouge. La Pologne disparaît des cartes et de nombreux polonais entrent en résistance contre l'occupant nazi. Georges participe activement au mouvement, sans que l'on en sache plus sur son rôle exact. De cette époque difficile date sans doute une culture du secret et du mystère qu'il conserve toute sa vie... Quoi qu'il en soit, il est fait prisonnier et déporté en Allemagne. II ne retrouve la liberté qu'à la veille de la libération, en mars 1945.

Après la guerre il fuit, comme beaucoup, l'occupation soviétique. Francophile et francophone, il émigre $d^{\prime}$ abord en Belgique, et fréquente l'Université libre de Louvain. Puis il se rend en France en 1947. II est admis à l'Institut d'optique, dont les locaux sont encore à Paris, boulevard Pasteur. II est diplômé en 1949, et rejoint le groupe de Maurice Françon [1913-1996] en tant que stagiaire du CNRS. Son seul diplôme d'ingénieur ne lui permet pas de préparer un doctorat, mais ses travaux et les résultats qu'il obtient lui permettent d'être recruté comme attaché de recherche puis de progresser rapidement au grade de maître de recherche en 1954.

Nomarski montre en microscopie des connaissances et une compréhension proprement exceptionnelles, débordant largement les informations habituelles des ouvrages ou enseignements classiques, et témoignant $d$ 'une formation non académique, peut-être acquise pendant la guerre. C'est donc tout naturellement qu' on lui confie l'enseignement de la microscopie à l'École supérieure d'optique à partir de 1953. Il fonde à la même époque le Laboratoire de microscopie de l'Institut d'optique, dont les activités abordent d'emblée tous les aspects de l'optique physique (diffraction, polarisation, interférences, photométrie), et qui s'étendront progressivement à toute l'optique instrumentale et à la spectrométrie.

\section{La microscopie à contraste interférentiel différentiel}

Les années qui suivent sont particulièrement fécondes. Rappelons le contexte : le néerlandais Frederik Zernike [1888-1966] a découvert le contraste de phase en 1933, une technique qu'il propose d'appliquer à la microscopie optique pour observer la structure interne des cellules sans coloration, donc en préservant leur intégrité. C'est une révolution conceptuelle, qui lance une course à l'innovation.

Le premier microscope à contraste de phase lou contraste interférentiel) voit le jour en 1942. D'autres systèmes sont imaginés et perfectionnés dans les années qui suivent. Les plus aboutis proposent $d$ 'introduire un dispositif interférentiel à l'intérieur du tube du microscope pour supprimer le phénomène de halo inhérent au contraste de phase. En 1947, F.H. Smith est le premier à utiliser des prismes de Wollaston pour la séparation et la recombinaison des faisceaux. Mais son système souffre a priori d'un inconvénient pratique majeur : le prisme utilisé pour la recombinaison des faisceaux doit être placé dans le plan focal de l'objectif, autrement dit dans l'objectif, un endroit généralement inaccessible. C'est à 
Nomarski que l'on doit l'innovation décisive : il utilise également des prismes de Wollaston, mais il en adapte la conception (un des cristaux est taillé obliquement à l'axe optique) pour externaliser le plan des franges et pouvoir placer le prisme à distance de l'objectif, par exemple dans la tourelle porte-objectif du microscope. La fabrication du dispositif est assez délicate, et est confiée à la petite entreprise J. Fichou. Son système s'impose définitivement à la communauté et donne à la microscopie à contraste de phase l'élan qui lui manque encore. Les premiers microscopes à contraste interférentiel différentiel, aussi appelés microscopes Nomarski, sont produits et commercialisés par la société Carl Zeiss dès 1965. Ils connaissent dès lors un essor considérable dans les laboratoires de recherche en raison de la qualité des images obtenues, aussi bien pour des systèmes en réflexion léclairage épiscopique) qu'en transmission (éclairage diascopique), s'imposant dans de nombreux domaines scientifiques et à travers le monde. Georges Nomarski est promu Directeur de recherche du CNRS en 1965. Il maintient des relations étroites avec la diaspora polonaise en Europe ou aux États-Unis, et se rend régulièrement à l'Institut d'optique appliquée de Varsovie à partir des années 1970, époque où il obtient la nationalité française.

\section{Les honneurs}

Ses travaux lui valent de nombreuses récompenses. II reçoit le prix du Duc d'Aumale décerné par l'Académie des sciences en 1952, le prix Gaumont de la Société d'encouragement pour l'industrie nationale en 1963 et le prix de la Microscopy Society de Chicago en 1970. Il est élu fellow de l'Optical Society of America en 1972, et obtient le glorieux titre d'honorary fellow de la Royal Microscopical Society de Londres. Il est également récipiendaire de la Abbe Medal, une récompense décernée par la State Microsco- pical Society of Illinois pour célébrer le $100^{\mathrm{e}}$ anniversaire de la théorie d'Abbe sur la formation d'images microscopiques.

Enfin, la SPIE (Society of Photo-optical Instrumentation Engineers) lui décerne en 1995 sa prestigieuse Gold Medal pour l'ensemble de sa carrière, et en hommage à l'impact de ses découvertes.

\section{Ultima verbale}

Georges Nomarski a contribué par l'invention du microscope à contraste interférentiel à l'émergence de nouvelles connaissances dans le secteur de la recherche fondamentale et appliquée. II prend sa retraite en 1980, mais maintient des relations avec le milieu de la recherche. Quoiqu'en proie à des soucis de santé, il honore volontiers des invitations à des conférences sur la microscopie. Il s'éteint à l'âge de 78 ans, à Antony, au sud de Paris, le 17 février 1997.

Ses anciens collègues et étudiants gardent le souvenir d'un homme d'une vaste culture scientifique, très créatif, qui a mis une grande énergie au développement de la technique du contraste interférentiel - parfois au détriment $d^{\prime}$ 'autres aspects de la vie d'un responsable d'équipe de recherche : il n'a ainsi encadré que peu de travaux de thèse et, bien que sa production intellectuelle (brevets et enveloppes Soleau) soit riche et variée, sa bibliographie scientifique est assez chiche.

Remerciements. L'auteur remercie chaleureusement Gérard Roblin pour son aide, et pour les informations inestimables qu'il a bien voulu lui communiquer.

\section{Référence}

[1] Maksymilian Pluta, Proceedings SPIE Vol. 1846, pp. 10-25 (1994).

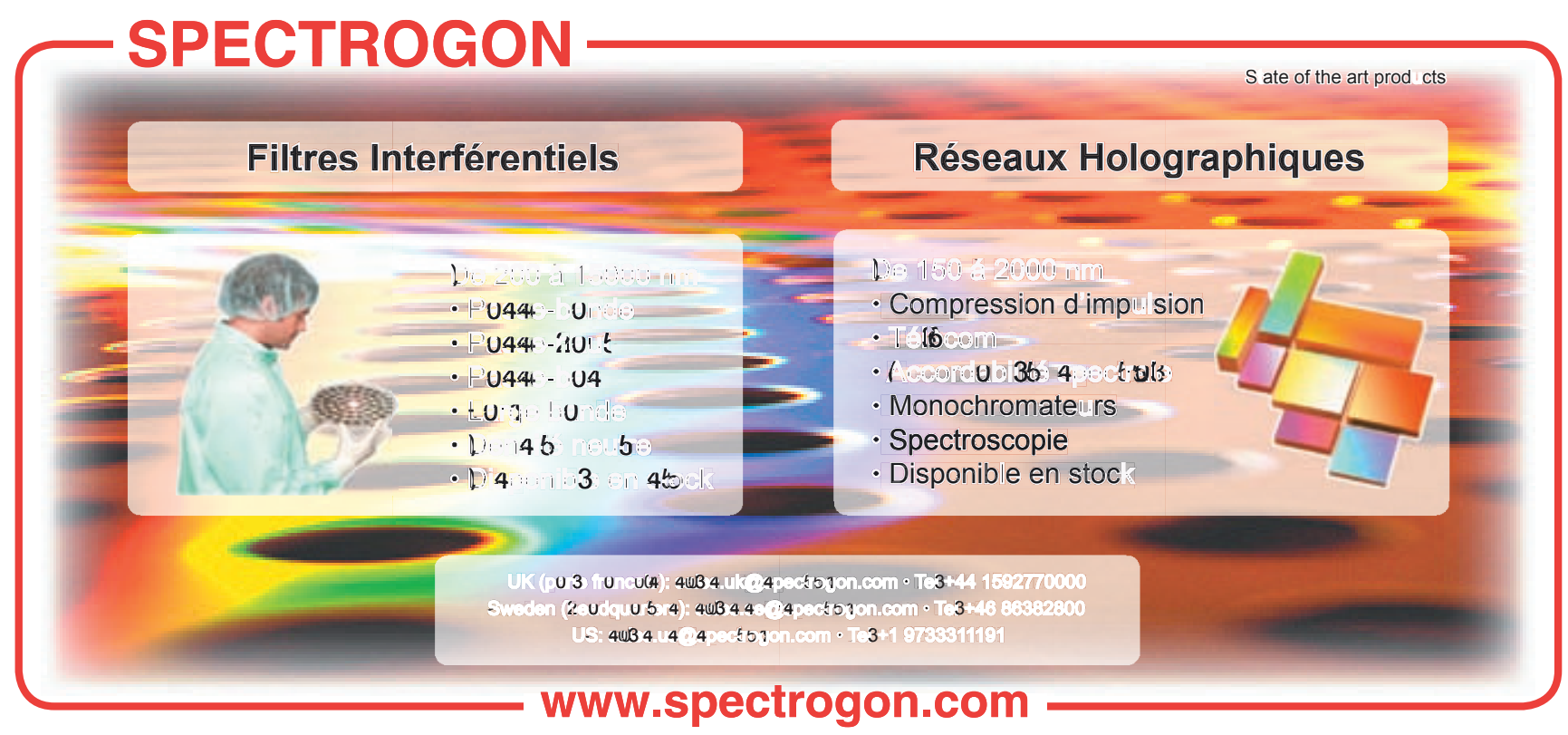

